\title{
SEMI-SOLID AGAR MEDIA FOR CULTURE AND DRUG SENSITIVITY TESTS OF TUBERCLE BACILLI FROM SPUTUM
}

\author{
BY \\ R. KNOX AND G. B. SKINNER \\ From the Department of Bacteriology, Guy's Hospital Medical School, Lordo:
}

(RECEIVED FOR PUBLICATION JANUARY 18, 1957)

Semi-solid agar media have been developed in this laboratory for the rapid culture and drug sensitivity testing of Mycobacterium tuberculosis (Knox, 1955 ; Knox, Swait, and Woodroffe, 1956 ; Knox and Woodroffe, 1957). It seemed that such media might be especially valuable in the examination of patients' sputa, and the present paper describes the results obtained in a small series of 50 specimens.

\section{Methods}

Method of Homogenizing Sputum.-Sputum specimens were homogenized using a modified Petroff's method. Sputum was treated as described by Mackie and McCartney (1953) except that after removing the supernatant fluid subsequent to centrifuging for half an hour at 3,000 r.p.m. the neutralized deposit was centrifuged further for $5 \mathrm{~min}$. at 1,000 r.p.m. This second spinning deposited the coarser particles of debris inseparable from sputum concentrates without removing all the tubercle bacilli. The supernatant fluid from this centrifuging was then used as an inoculum after neutralization. It was always inoculated upon the day of homogenization.

Media.-Kirchner or Fisher medium containing $0.125 \%$ agar and $10 \%$ horse serum was used as already described (Knox, 1955; Knox et al., 1956). The agar was heated to melt it and was then cooled to $48^{\circ} \mathrm{C}$. While it was still liquid the horse serum and penicillin ( 50 units $/ \mathrm{ml}$.) were added to it. It was then dispensed in $2 \mathrm{ml}$. amounts into 6 in. $\times 5 / 8$ in. tubes and still kept at $48^{\circ} \mathrm{C}$. For drug sensitivity tests, $0.2 \mathrm{ml}$. amounts of solutions of isoniazid, streptomycin, or para-amino-salicylic acid (P.A.S.) were added to give the required final concentration.

Inoculation and Culture.-Tubes were inoculated using Pasteur pipettes and were then tightly sealed with rubber bungs and allowed to cool to $37^{\circ} \mathrm{C}$. The tubes were incubated at $37^{\circ} \mathrm{C}$. and inspected at two- or three-day intervals. We have used the viewing box previously described (Knox et al., 1956), but any similar system giving suitable lighting and magnification up to $\times 6$ is satisfactory. The tubes were photographed as described elsewhere (Engel and Knox, 1957).

Direct Sensitivity Tests.-For direct sensitivity tests one drop of homogenized sputum was inoculated into tubes prepared as described above. For each specimen, a drug-free control tube was also inoculated. Drug-free media were also inoculated with a 1 in 10 , 1 in 100 , and 1 in 1,000 dilution of each sputum homogenate to give an estimate of the number of viable organisms it contained.

Indirect Sensitivity Tests.-As soon as adequate growth was obtained in one of the drug-free tubes, an indirect sensitivity test was performed. A colony of about $0.5 \mathrm{~mm}$. in diameter was removed from the agar by Pasteur pipette and delivered into $0.5 \mathrm{ml}$. of distilled water in a bijou bottle containing a small quantity of Ballotini beads. The colony was broken up by being shaken for five minutes in a mechanical shaker and the suspension was then used as an inoculum with no further treatment. If only a small quantity of material was available for cultivation it was found preferable not to put up direct sensitivity tests but to use all the specimen in the drug-free tubes and then to use growth in these for indirect sensitivity estimations. With both direct and indirect sensitivity tests a parallel set of tubes was inoculated with the H37Rv strain of $M$. tuberculosis.

\section{Results}

The sputa all came from patients who had had tubercle bacilli in films of immediately preceding specimens.

Cultures were performed on 60 specimens and were successful in 50 of these. Table I shows that

TABLE I

PRIMARY CULTURE OF M. TUBERCULOSIS FROM 50 SPUTUM HOMOGENATES

\begin{tabular}{l|c|c}
\hline Week & No. Positive & Cumulative (\%) \\
\hline 1st & $16(32 \%)$ & 32 \\
2nd & $19(38 \%)$ & 70 \\
3rd & $10(20 \%)$ & 90 \\
4th & $5(10 \%)$ & 100 \\
\hline
\end{tabular}


positive cultures were obtained from $32 \%$ of these in the first week after inoculation, from $70 \%$ within two weeks, and from $90 \%$ in less than three weeks. The average time taken for the appearance of growth recognizable by the naked eye was 12 days.

Direct sensitivity tests in semi-solid agar were performed on 17 of these 50 specimens. Table II gives the times at which results were first readable in these 17 specimens. Some typical results are shown in Fig. 1.

TABLE II

PRIMARY DRUG SENSITIVITY TESTS ON 17 SPUTUM HOMOGENATES

\begin{tabular}{c|c|c}
\hline Week & $\begin{array}{c}\text { No. of Tests } \\
\text { Readable }\end{array}$ & $\begin{array}{c}\text { Cumulative } \\
(\%)\end{array}$ \\
\hline 1st & $1(6 \%)$ & 6 \\
2nd & $10(59 \%)$ & 65 \\
3rd & $2(12 \%)$ & 76 \\
4th & $3(18 \%)$ & 94 \\
5th & $1(6 \%)$ & 100 \\
\hline
\end{tabular}

In 11 specimens single colonies were removed from primary sputum cultures and shaken with Ballotini beads as described above. Indirect sensitivity tests were performed with this material. The average period of incubation for an indirect sensitivity test in these cases was seven days.
Twenty-four sputum homogenates were inoculated directly both on Löwenstein-Jensen slopes and into semi-solid Kirchner agar. From three $\overrightarrow{\vec{s}}$ of these specimens tubercle bacilli were not iso-o lated in either medium. Of the 21 specimens which yielded tubercle bacilli, three gave a posi- $\overline{\bar{c}}$. tive result in semi-solid agar only, two on Löwen- $\overparen{\mathbb{Q}}$ stein-Jensen medium only, and 16 on both media. D The average time taken for recognizable growth ${ }^{\infty}$ to appear from these specimens was 13.5 days in $\vec{O}$ the semi-solid medium and 23.5 days for the same $\overrightarrow{\vec{H}}$ specimens on Löwenstein-Jensen medium.

The characteristic features of the growth of $M$. 횽 tuberculosis in semi-solid Kirchner, Fisher, and $\vec{\circ}$ Dubos agar have been fully described (Knox et $\stackrel{\circ}{\circ}$ al., 1956). All these media are reasonably satis- $\dot{\omega}$ factory for drug sensitivity tests (Knox and Wood- $\mathcal{Y}$ roffe, 1957), but for primary culture and sensi-응 tivity tests directly from sputum we have used Kirchner rather than Dubos semi-solid agar be- $z$ cause it gives more rapid growth. The typically cream-coloured, craggy, and crumb-like colonies 3 in this medium can easily be distinguished from sputum debris and from the rare contaminants which survive in the presence of penicillin. Hove $\vec{E}$ ever, it has sometimes been hard to differentiate. very early growth from sputum debris, everg

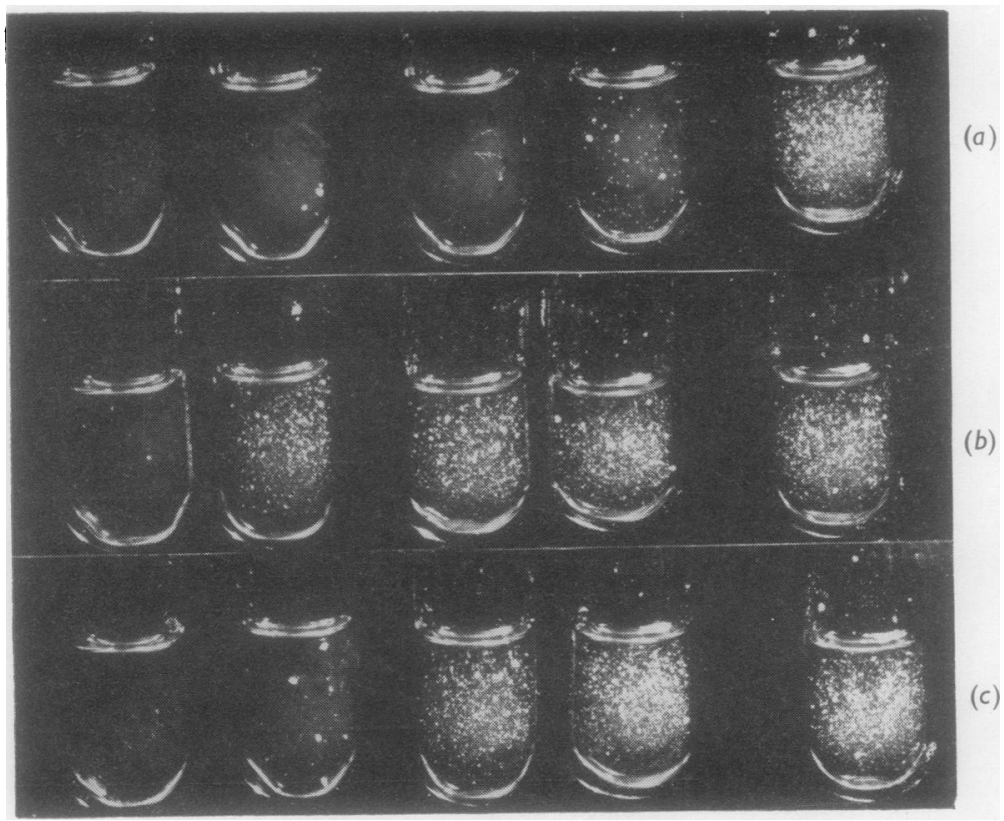

Figs. 1, 2, and 3-Photographs of tubes containing semi-solid Kirchner agar? incubated for 14 days after inoculation. Each row consisted of tubes containing (from left to right) 100,0 10,1 , or $0.1 \mu \mathrm{g} . \mathrm{ml}$. of drug with a drug-free control on the right. In $D$ each figure the top row $(a)$ contained음 isoniazid, the middle $(b)$ streptomycin, and the lower (c) P.A.S. N

FIG. 1.-Tubes inoculated directly with sputum concentrate from Patient B. Strain sensitive (apart from a few colonies) to isoniazid, $0.1 \mu \mathrm{g} . / \mathrm{ml}$, but resistant to streptomycin, 10, and P.A.S., $1, \mu \mathrm{g} . / \mathrm{ml}$. 


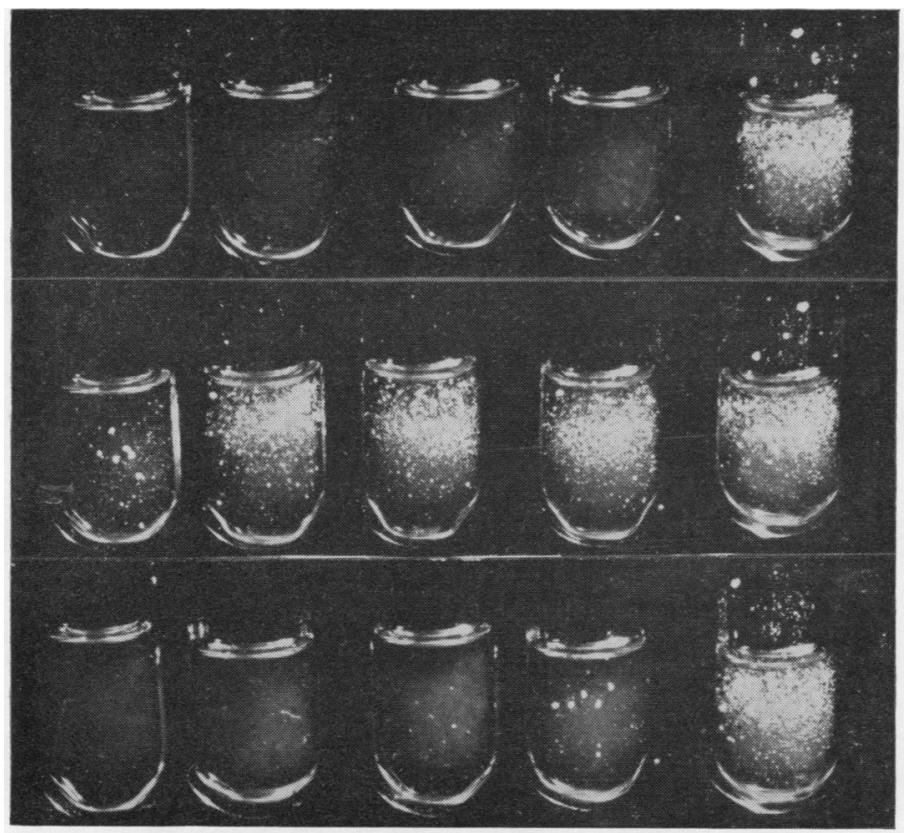

(a)

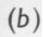

Fig. 2. - Tubes inoculated directly with sputum concentrate from Patient P. Strain sensitive to isoniazid, 0.1, and (apart from a few colonies) P.A.S., $0.1 \mu \mathrm{g} . / \mathrm{ml}$., but fully resistant to streptomycin, 10 , and partly to $100 \mu \mathrm{g} . / \mathrm{ml}$.

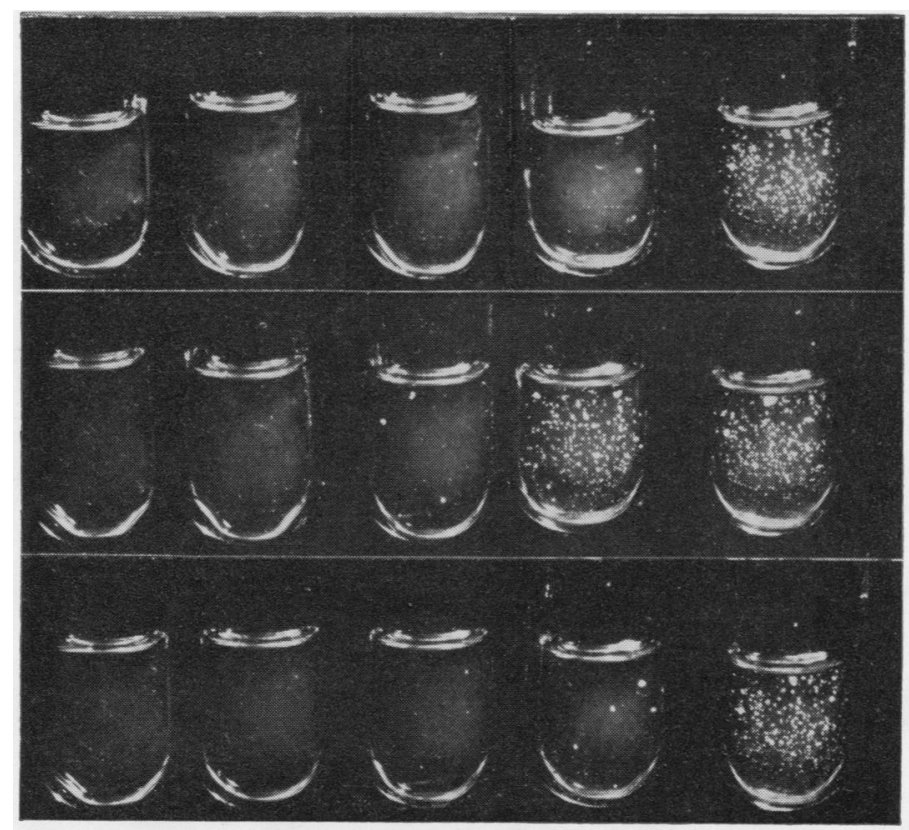

(a)

FIG. 3.-H37Rv strain for comparison, sensitive (apart for a few colonies) to isoniazid, 0.1 , streptomycin, 1 , and P.A.S., $0.1 \mu \mathrm{g} . / \mathrm{ml}$. 
though the additional light centrifugation does remove gross debris.

In an attempt to overcome this difficulty tetrazolium salt was added to the medium for primary culture though not for drug sensitivity tests. At first triphenyl-tetrazolium chloride was used, but as the result of a suggestion by Dr. I. Tsuchiya (personal communication, 1956) neotetrazolium chloride $(0.002 \%)$ is now being used. The neotetrazolium salt gives purple colonies which can be clearly distinguished from sputum debris in as little as eight days. The purple colour with neotetrazolium appears earlier than the pink given by triphenyl tetrazolium, and the neotetrazolium dye is also considerably less toxic to the tubercle bacilli in equivalent concentrations.

\section{Discussion}

Pryce (1941) in his paper on the slide cultivation of tubercle bacilli observed that the detection of the organisms was very much a matter of magnification. Slide methods have been used successfully to obtain growth from sputum between six and 14 days with an average of 10 days (Reed, 1953, 1954), but the necessary microscopical examination of slides is time-consuming, and the transferring of slides bearing dried sputum into culture media is dangerous and in the authors' experience is also attended by a high contamination rate. With semi-solid agar media and with a method of inspecting cultures with a hand lens and suitable illumination, it has been possible to achieve primary isolation and sensitivity tests in times which compare favourably with any other method. All the tubes for primary culture, primary sensitivity test, and viable count can be kept together and quickly inspected at frequent inter- vals in an ordinary 24-tube rack. They occupy little space in incubators and can be easily trans- $\vec{F}$ ported. Apart from an efficient means of view- $\stackrel{\rho}{?}$ ing early growth, no special equipment is required.

The results described here are clearly only of a $\frac{\bar{\omega}}{\frac{5}{6}}$. preliminary nature. It is not claimed that the $\vec{\mathbb{}}$ exact media used here are necessarily the best. $\frac{2}{0}$ The purpose of this paper is to show that semi- $\omega$ solid agar media give a safe, quick method for $\overrightarrow{0}$ culturing and testing the drug sensitivity of $\vec{H}$ tubercle bacilli, demanding no special equipment $\bar{\sigma}$ and worthy of more extended trial for routine use.

\section{Summary}

Semi-solid agar media have been successfully used for primary culture and drug sensitivity tests $V$ of tubercle bacilli directly from sputum homo-음 genates. In 50 specimens from which cultureswere obtained, a positive result was readable in $Z$ 35 within two weeks and in 45 within three weeks. On 17 specimens on which direct sensitivity tests 3 were performed, results were readable in 13 within three weeks. The addition of penicillin $(50 \overrightarrow{\vec{\theta}}$ units $/ \mathrm{ml}$.) greatly reduced contamination, whige or the addition of neotetrazolium chloride $(0.002 \%)$. made it easy to differentiate the purple colonis of $M$. tuberculosis from debris in primary culture from sputum.

\section{REFERENCES}

Engel, C. E., and Knox, R. (1957). Nature (Lond.), 179, 411.

Knox, R. (1955). Lancet, 2, 110.

- and Woodroffe, R. (1957). J. gen. Microbiol., 16, 647.

- Swait, E., and Woodroffe, R. (1956). Ibid., 15, 359.

Mackie, T. J., and McCartney, J. E. (1953). Handbook of Practical Bacteriology, 9th ed., p. 404. Livingstone, Edinburgh.

Pryce, D. M. (1941). J. Path. Bact., 53, 327.

Reed, R. W. (1953). Canad. J. med. Sci., 31, 367.

- (1954). Canad. J. Microbiol., 1, 30.

Tsuchiya, I. (1956). Personal communication. 